Gynäkologische Endokrinologie 2009 · 7:129 DOI 10.1007/s10304-009-0323-8

Online publiziert: 28. August 2009

๑) Springer Medizin Verlag 2009

\author{
W. Urdl ${ }^{1} \cdot$ T. Strowitzki ${ }^{2}$ W. Küpker ${ }^{3}$ \\ ${ }^{1}$ Geburtshilflich-Gynäkologische Universitätsklinik Graz \\ ${ }^{2}$ Abt. für Gynäkologische Endokrinologie und Fertilitätsstörungen, \\ Universitätsfrauenklinik, Heidelberg \\ ${ }^{3}$ Endometriosezentrum, Stadtklinik Baden-Baden
}

\title{
Störungen der Geschlechtsentwicklung und Geschlechtsidentität
}

piemaßnahmen, insbesondere jedoch als Entscheidungsgrundlage für korrigieren$\mathrm{de}$, chirurgische Interventionen sind in derartigen Fällen die Ergebnisse einer interdisziplinären Meinungsbildung von Kinderund Jugendendokrinologen, Kinderchirurgen, Kinderurologen, Psychologen, Kinderund Jugendpsychiatern, Gynäkologen, Genetikern und ggf. Neonatologen, Sozialarbeitern und Medizinethikern maßgeblich.

Können bei Personen mit Störungen der pränatalen somatosexuellen Differenzierung und ambivalentem Genitalbefund im Zuge der weiteren Entwicklung in Kindheit und Adoleszenz Störungen der Geschlechtsidentität nachweisbar werden, sind von derartigen Phänomenen Störungen der Geschlechtsidentität zu trennen, die als „Transsexualität“ bezeichnet werden (s. Beitrag von H.A.G. Bosinski). Hierbei handelt es sich um Störungen, die beiderlei Geschlechter betreffen können und durch eine profunde Irritation der Geschlechtsidentität charakterisiert sind. Diese Menschen, die in der Regel einen ihrem Genotyp zugehörigen Phänotyp mit entsprechenden, unauffälligen Genitalien aufweisen, fühlen sich dem ihrer Biologie und Genetik konträren Geschlecht zugehörig und empfinden eine Aversion gegenüber ihrer eigenen Geschlechtsausstattung, die sie häufig veranlasst, zwanghaft den Wunsch nach hormoneller und chirurgischer Geschlechtsumwandlung zu äußern.

Im Beitrag von T.O. Nieder und H. Richter-Appelt wird umfassend auf aktuelle Begriffsbestimmungen, diagnostische Kriterien und über Erfahrungen, die Lebensbegleitung transsexueller Personen aus psychosozialer, psychotherapeutischer und gynäkologischer Sicht betreffend, eingegangen.
Schließlich werden „Behandlungsgrundsätze bei Transsexualität" ausführlich von W. Urdl dargestellt. Im Besonderen wird in diesem Beitrag auf die Sinnhaftigkeit der Einhaltung etablierter Betreuungs- bzw. Behandlungsstrategien, wie z. B. auf die Einhaltung 5 zeitlich aufeinanderfolgender Phasen, bestehend aus - Diagnostik und Differenzialdiagnostik, - Alltagstest,

- gegengeschlechtliche Hormontherapie,

- Transformationschirurgie,

- Nachbetreuung,

hingewiesen.

Wir glauben, Ihnen mit diesem Heft aktuelle und interessante Übersichten, die Themen „gestörte Geschlechtsentwicklung " und "gestörte Geschlechtsidentität" („Transsexualität") betreffend, anbieten zu können, und würden uns freuen, Ihnen mit der Lektüre dieser Beiträge Anregungen für Ihre Tätigkeit in Klinik und Praxis zu vermitteln.

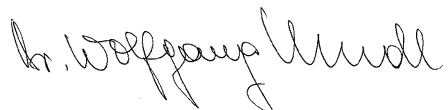

Prof. Dr. Wolfgang Urdl

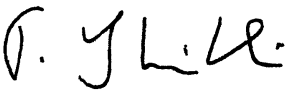

Prof. Dr. Thomas Strowitzki

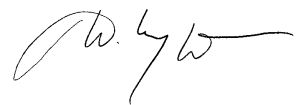

Prof. Dr. Wolfgang Küpker

\section{Korrespondenzadresse}

Prof. Dr.W. Urdl

Geburtshilflich-Gynäkologische Universitätsklinik Graz

Auenbruggerplatz 14, $8036 \mathrm{Graz}$

Österreich

wolfgang.urdl@meduni-graz.at 